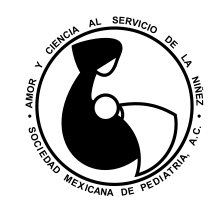

ARTíCULO DE REVISIÓN

Vol. 86, No. 4 • Julio-Agosto 2019

pp 162-164

REDIATRÍA

Recibido: 15/10/2018

Aceptado: 06/04/2019

dx.doi.org/10.35366/SP194H

\title{
Escalas predictoras para identificar vía aérea difícil en población pediátrica: su utilidad en el servicio de urgencias
}

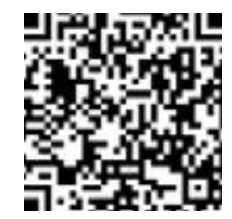

\author{
Flavio Figueroa-Uribe, ${ }^{1, *}$ Jorge Omar Flores-del Razo, ${ }^{2}$ Valentina Vega-Rangel, ${ }^{2}$ \\ Víctor Méndez-Trejo, ${ }^{3}$ Maribel Ferrer-López, ${ }^{3}$ Norma Araceli González-Chávez ${ }^{4}$ \\ ${ }^{1}$ Hospital Pediátrico de Peralvillo; ${ }^{2}$ Instituto Nacional de Pediatría; ${ }^{3}$ Hospital General de Cuautitlán "Gral. José Vicente \\ Villada”, Instituto de Salud del Estado de México; ${ }^{4}$ Hospital de Especialidades “Dr. Belisario Domínguez”, México.
}

\begin{abstract}
RESUMEN
En este artículo se describen de forma sucinta las escalas predictoras existentes de vía aérea difícil y su posible aplicabilidad en pacientes pediátricos en el servicio de urgencias. De manera particular se mencionan cuatro escalas Mallampati, Patil-Aldreti (distancia tiroideo-mentoniana), extensión de la articulación atlanto-occipital y la distancia esterno-mentoniana. En general, ninguna de las escalas predice con certeza si habrá dificultad para la intubación, de ahí que es fundamental el monitoreo en el manejo de todo paciente que será sometido a intubación, especialmente aquéllos con sospecha de vía aérea difícil, al utilizar cualquiera de las escalas.
\end{abstract}

Palabras clave: Vía aérea difícil, escalas predictoras, pediatría.

\section{INTRODUCCIÓN}

La American Society of Anesthesiologists (ASA) define como vía aérea difícil la existencia de factores clínicos que complican la ventilación administrada por una mascarilla facial o la intubación realizada por una

\footnotetext{
*Correspondencia: FFU, tbcv_al@hotmail.com

Conflicto de intereses: Los autores declaran que no tienen. Citar como: Figueroa-Uribe F, Flores-del Razo JO, Vega-Rangel V, Méndez-Trejo V, Ferrer-López M, GonzálezChávez NA. Escalas predictoras para identificar vía aérea difícil en población pediátrica: su utilidad en el servicio de urgencias. Rev Mex Pediatr. 2019; 86(4):162-164.

[Predictive scales to identify difficult airway in the pediatric population: usefulness in the Emergency Department]
}

\begin{abstract}
This article succinctly describes the existing difficult airway predictive scales and their possible applicability in pediatric patients in the emergency department. In particular, four Mallampati, Patil-Aldreti (thyroid-chin distance), extension of the atlanto-occipital joint, and the sterno-chin distance are mentioned. In general, none of the scales predicts with certainty if there will be difficulty for intubation. Therefore, it is essential to monitor all patients who are going to be subjected to intubation, especially those detected through these scales with suspected difficult airway.
\end{abstract}

Keywords: Difficult airway, predictive scales, pediatrics.

persona experimentada. La ventilación difícil se define como la incapacidad del personal entrenado para mantener la saturación de oxígeno por arriba de $90 \%$ usando una mascarilla facial, con una fracción inspirada de oxígeno de $100 \%$. La intubación difícil se define como la necesidad de tres o más intentos para la intubación de la tráquea o más de 10 minutos para conseguirla.

Es trascendental recordar que a mayor grado de dificultad de la vía aérea, mayor incidencia y severidad de las complicaciones.

Las complicaciones relacionadas con el manejo de la vía aérea pediátrica son frecuentes y en ocasiones generan morbilidad y mortalidad, afectando principalmente a recién nacidos y lactantes menores. Dentro de la morbilidad puede ocurrir ventilación inadecuada, 
apnea y obstrucción bronquial. La mayor parte de estas complicaciones son evitables y al analizarse se detectan los siguientes problemas: falla en reconocer o anticiparse a los problemas, revisión inadecuada de equipo y monitores, escasa vigilancia, preparación insuficiente frente a situaciones adversas detectadas y falta de habilidades técnicas, especialmente en situaciones de estrés.

Los pacientes pediátricos presentan un amplio espectro de enfermedades, tanto congénitas como adquiridas, que pueden repercutir en la vía aérea, dificultando la intubación o ventilación. Para optimizar el manejo de una vía aérea difícil, es importante comprender las diferencias anatómicas de la vía aérea pediátrica, así como familiarizarse con las enfermedades y síndromes comunes que la afectan.

El manejo de la vía aérea y el mantenimiento de una adecuada oxigenación son objetivos centrales en todo paciente que ingresa al servicio de urgencias. Si se enfrenta una vía aérea difícil, se puede llegar a una situación de emergencia, con consecuencias deletéreas para el paciente.

Son escasos los estudios de la vía aérea realizados en urgencias y en especial los que analizan factores predictores de dificultad. La mayoría de ellos se han hecho en salas de anestesiología, otros en unidades de cuidado intensivo y algunos de ellos en escenarios de simulación.

Los pacientes con vía aérea difícil pueden dividirse en dos grupos: aquéllos con patología obvia (tumores, abscesos, deformidades congénitas, etcétera) y aquéllos sin signos evidentes de vía aérea difícil. Los primeros son relativamente fáciles de reconocer.

La historia clínica y el examen físico ayudan a identificar a los pacientes con vía aérea difícil. Ninguna de las clasificaciones que se mencionan más adelante predicen con certeza si la intubación tendrá o no complicaciones, pues la intubación endotraqueal está en función de factores anatómicos diversos. No obstante, las escalas son de utilidad en el quehacer diario de todo médico, ya que es mejor prever una vía aérea difícil, en lugar que se presente de forma inesperada. Un aspecto a señalar es que las escalas que a continuación se describen no se crearon de manera específica para la edad pediátrica.

\section{Escala de Mallampati}

Se evalúa mediante la visualización de las estructuras anatómicas faríngeas, con el paciente en posición sedente y con la boca completamente abierta. Para la detección de vía aérea difícil se ha reportado sensibi- lidad de $60 \%$, especificidad de $70 \%$ y valor predictivo de acierto de $13 \%$.

Se divide en cuatro clases:

- Clase I. Visibilidad del paladar blando, úvula y pilares amigdalinos.

- Clase II. Visibilidad del paladar blando y úvula.

- Clase III. Visibilidad del paladar blando base de la úvula.

- Clase IV. Imposibilidad para ver el paladar blando.

$$
\begin{gathered}
\text { Escala de Patil-Aldreti } \\
\text { (distancia tiroideo-mentoniana) }
\end{gathered}
$$

Evalúa la distancia que existe entre el cartílago tiroides (escotadura superior) y el borde inferior del mentón, en posición sedente, cabeza extendida y boca cerrada. Tiene sensibilidad de $60 \%$, especificidad de $65 \%$, predicción de $15 \%$.

- Clase I. Más de 6.5 cm (laringoscopía e intubación endotraqueal probablemente sin dificultad).

- Clase II. De 6.0 a $6.5 \mathrm{~cm}$. (laringoscopía e intubación endotraqueal con cierto grado de dificultad).

- Clase III. Menos de $6.0 \mathrm{~cm}$. (intubación endotraqueal muy difícil o imposible).

\section{Extensión de la articulación atlanto-occipital}

En esta prueba, el cuello se flexiona moderadamente $\left(25^{\circ}-30^{\circ}\right)$, y la articulación atlanto-occipital se extiende, alineando así los ejes oral, faríngeo y laríngeo en "posición de olfateo matutino". Una persona normal puede extender su articulación atlanto-occipital hasta $35^{\circ}$.

Grados de reducción de la extensión de la articulación atlanto-occipital en relación con los $35^{\circ}$ de normalidad:

- Grado I. Si no hay límites para extender la cabeza $\left(35^{\circ}\right)$.

- Grado II. Si la extensión se limita en un tercio de su valor normal $\left(22^{\circ}\right)$.

- Grado III. Si la extensión se limita a dos tercios de su valor normal $\left(15^{\circ}\right)$.

- Grado IV. Si no se puede extender la cabeza $\left(0^{\circ}\right)$.

\section{Distancia esterno-mentoniana}

Evalúa la distancia de una línea recta que va del borde superior del manubrio esternal a la punta del mentón, 
manteniendo la cabeza en completa extensión y la boca cerrada. Se ha estimado su sensibilidad en $80 \%$, especificidad de $85 \%$ y valor predictivo positivo de $27 \%$.

- Clase I. Más de $13 \mathrm{~cm}$.

- Clase II. De 12 a $13 \mathrm{~cm}$.

- Clase III. De 11 a $12 \mathrm{~cm}$.

- Clase IV. Menos de $11 \mathrm{~cm}$.

\section{Prueba evaluativa de Cormack y Lehane}

Durante la intubación se realiza una evaluación del grado de dificultad y se clasifica al paciente en una de las siguientes categorías:

- Grado I. Se observa el anillo glótico en su totalidad (intubación muy fácil).

- Grado II. Sólo se observa la comisura o mitad posterior del anillo glótico (cierto grado de dificultad).

- Grado III. Sólo se observa la epiglotis sin visualizar orificio glótico (intubación muy difícil pero posible).

- Grado IV. Imposibilidad para visualizar incluso la epiglotis (intubación sólo posible con técnicas especiales).

\section{BIBLIOGRAFÍA RECOMENDADA}

1. Orozco E, Álvarez J, Ornelas JM, Predicción de intubación difícil mediante escalas de valoración de la vía aérea. Rev Anestesiol Mex. 2011; 78(5): 393-399.

2. Echeverry PC, Engelhardt T. Algoritmo para el manejo de la vía aérea difícil en pediatría. Rev Colomb Anestesiol. 2014; 5 (2): 19-31.
3. Vieira AP, Andrade TL, Lauzi J, Watanabe M. Intubación difícil en niños: aplicabilidad del índice de Mallampati. Rev Bras Anestesiol. 2011; 61 (2): 84-87.

4. Mancera $\mathrm{G}$, Arenas $\mathrm{AD}$. La vía aérea difícil y sus implicaciones en pediatría. Anestesia Pediátrica e Neonatale. 2009; 7 (2): 1-24.

5. Osses H. Vía aérea difícil en pediatría. Rev Chil Anest. 2010; 39 : 125-132.

6. Andreu E, Schmucker E, Drudis R. Algoritmo de la vía aérea difícil en pediatría. Rev Esp Anestesiol Reanim. 2011; 58: 304-311.

7. Ríos MA, Gómez LM, Aguirre OO. La vía aérea pediátrica: Algunos conceptos para tener en cuenta en el manejo anestésico. Rev Colomb Anestesiol. 2011; 40 (3): 199-202.

8. Kunze NS. Análisis de algoritmos de manejo de vía aérea difícil en pediatría. Rev Chil Anest. 2009; 38: 91-100.

9. Ríos GE, Reyes CJ. Valor predictivo de las evaluaciones de la vía aérea difícil. Asoc Mex Med Cir Trauma. 2008; 8 (3): 63-70.

10. Avelar SB, Rangel AF, Bosques NG. Evaluación preoperatoria para intubación en pacientes pediátricos combinando valores predictivos de dos clasificaciones clínicas. Rev Mex Anestesiol. 2009; 32 (2): 89-96.

11. Jiménez HA, Pardo DE, Alarcón J. Manejo de la vía aérea pediátrica. Revista Gastrohnup. 2015; 17 (3): S38-S50.

12. Salazar CB. Evaluación de la vía aérea difícil. Rev Med Costa Cen. 2011; 68 (597): 241-245.

13. Oriol LM, Hernández MM, Hernández BC, Álvarez FA. Valoración, predicción y presencia de intubación difícil. Rev Mex Anest. 2009; 32 (2): 41-49.

14. Valero R, Mayoral V, Massó E. Evaluación y manejo de la vía aérea difícil prevista y no prevista. Adopción de guías de práctica. Rev Esp Anestesiol Reanim. 2008; 55: 563-570.

15. Chavarría I, Robles B, Loria C. Complicaciones agudas por intubación orotraqueal en la sala de urgencias. Arch Med Urgencias Mex. 2012; 4 (1): 20-25.

16. Meléndez $\mathrm{H}$, Leal $\mathrm{D}$, Concordancia de la evaluación objetiva y subjetiva en la predicción y hallazgo de la vía aérea difícil. Rev Colomb Anestesiol. 2010; 38 (1): 34-39. 